Materials and Methods A quasi-experimental/retrospective study was carried out, analysing discrepancies between chronic medicines and drugs prescribed in the hospital, before and after a medicines reconciliation programme was implemented.

Patients admitted into a general surgery unit for more than $24 \mathrm{~h}$ who were taking $\geq 3$ drugs chronically at home were included.

A standardised interview was conducted to record chronic medicines. Pharmacists detected and investigated discrepancies. The severity of unintended discrepancies was assessed by consensus with medical staff using the National Coordinating Council for Medication Error Reporting and Prevention 2001 classification. A computerised reconciliation tool, integrated into the electronic prescription, was implemented during the intervention phase.

Results A total of 191 patients were included (52.9\% male, 47.1\% female), 107 patients in the phase before intervention and 84 in the phase after intervention.

1,951 drugs were investigated, and 1,678 discrepancies were detected. There were 167 unintended discrepancies, 102 (10.6\% of drugs investigated) in the first phase and 65 in the second phase $(6.6 \%), p=0.0021$. Omission of drugs was the most common unintended discrepancy, being $89(9.2 \%)$ in the phase before and $55(5.6 \%)$ in the phase after intervention, $p=0.0027$

Unintended discrepancies were grade C severity in $79.2 \%$ of those detected, decreasing in the second phase $(3.95 \%$ of total drugs investigated) compared to the first one (8.61\%), $p<0.05$.

Conclusions The implementation of the medicines reconciliation programme has shown a reduction of the rate of unintended discrepancies detected during admission into a general surgery unit. Omission of drugs was the most common type of discrepancy detected in both phases and decreased after intervention.

No conflict of interest.

\section{GRP-088 IMPACT OF THE PHARMACEUTICAL VALIDATION OF PRESCRIPTIONS FOR INPATIENTS WITH RENAL IMPAIRMENT}

doi:10.1136/ejhpharm-2013-000276.088

A Paula, S Buendía, I Marquinez, A Ribed, ME Durán, M Sanjurjo. Hospital Gregorio Marañon, Pharmacy, Madrid, Spain

Background The use of drugs in patients with nephropathy carries certain risks. Therefore, dosages must be adjusted.

Purpose To describe pharmaceutical interventions (PIs) on electronic prescriptions for patients with renal impairment $(\mathrm{RI}=$ creatinine clearance $<50 \mathrm{ml} / \mathrm{min}$ ) admitted from emergencies.

Materials and Methods Nine-month observational study performed with patients with RI admitted from emergencies to wards with electronic prescribing. Glomerular filtration rate was calculated with MDRD-4 IDMS. Treatments were reviewed to evaluate the suitability of doses using the data sheets Medimecum, Micromedex and Lexicomp. If the dose was not correct, a PI was written in the 'Alerts' section of the prescribing programme which was subsequently seen by the physician. Demographics, date of the PI, serum creatinine, creatinine clearance, drug, PI, acceptance or rejection and why and evolution of renal function on the seventh day of the acceptance were recorded in the database.

Results 5311 patients were included, 221 PIs were made for 181 patients $(3.41 \%)$. Patients for whom interventions were made had a mean age of 78 (29-102) and $49.2 \%$ were male. The drug with most interventions was levofloxacin (29.9\%). The PIs were: dose-related $(65.6 \%)$, increase of therapeutic range $(26.7 \%)$ and contraindication (7.2\%). 65.6\% were accepted. The clinical consequences after acceptance of the PI were: improved renal function (54.5\%), deteriorated $(12.4 \%)$, unchanged $(11.0 \%)$ or not evaluable $(22.1 \%)$. In patients whose PI was rejected, renal function improved in $57.63 \%$, deteriorated in $16.95 \%$, was unchanged in 6.78 and not evaluable in $18.64 \%$.
A Chi-square test was applied to study whether the evolution of renal function depended on acceptance ( $p$ value 0.634 ).

Conclusions Electronic prescribing is a useful tool for identifying opportunities for PI in patients with RI. Differences in renal function progression between the group in which the PI were accepted and the group in which these were rejected were not statistically significant.

No conflict of interest.

\section{GRP-089 IMPLEMENTATION OF A "MEDICATION SAFETY" CURRICULUM AS PART OF THE CONTINUING EDUCATION PROGRAMME FOR PHARMACISTS}

doi:10.1136/ejhpharm-2013-000276.089

${ }^{1} \mathrm{G}$ Picksak, ${ }^{2 P}$ Kantelhardt, ${ }^{3} \mathrm{M}$ Hug. 'Hannover Medical School, Central Pharmacy, Hannover, Germany; 'University Medical Center of the Johannes Gutenberg University Mainz, Department of Neurosurgery, Mainz, Germany; ${ }^{3}$ University Medical Center Freiburg, Pharmacy, Freiburg, Germany

Background The 'action plan for the improvement of medication safety' issued by the German ministry of health demands a culture of safety awareness. To achieve this goal, an emphasis on medication safety should be placed in the education of health care professionals. In this context the German Society of Hospital Pharmacists (ADKA) has developed a curriculum on medication safety.

Purpose A workshop has been developed to improve the awareness of health care professionals regarding medication errors and the risks involved. The tools allow the pharmacist to perform a selfcontained failure analysis as a basis for a goal-oriented prevention strategy.

Materials and Methods The curriculum consists of three parts. After a brief introduction, the tools to develop strategies for error prevention are explained. These tools are then applied to real life examples of medication errors in the clinical routine or in the community pharmacy respectively. The curriculum has been presented to the local boards of pharmacy and the association of statutory health insurance physicians.

Results After approval by the board of pharmacy of Lower Saxony, a pilot course was conducted. Within four days of the first invitation being sent, almost 30 participants had enrolled. Finally more than 50 participants, the majority of whom were community pharmacists successfully completed the curriculum, which was evaluated by the local board of pharmacists.

Conclusions The rapid and strong response to the invitation is a sign that the subjects 'medication safety and medication errors' are of particular interest to community pharmacists. It also tells us that medication safety is not a substantial part of continuing education. An evaluation has shown that the time allotted for the curriculum (90 min.) is apparently too short and should be extended to at least $150 \mathrm{~min}$. The participants appreciated the opportunity to develop their own strategies to prevent medication errors. The experience accumulated so far demonstrates that the basic concept of the curriculum, now available to all interested boards of pharmacists, is a promising strategy.

No conflict of interest.

\section{GRP-090 IMPLEMENTATION OF GRAVIMETRIC ANALYSIS IN THE PHARMACY DEPARTMENT}

doi:10.1136/ejhpharm-2013-000276.090

V Gimeno Ballester, I Larrodé, M Uriarte, 0 Pascual, JM Real, MJ Agustín, P Palomo, MR Abad. Universitary Hospital Miguel Servet, Pharmacy, Zaragoza, Spain

Background Parenteral nutrition (PN) involves multicomponent intravenous mixtures of high complexity and is considered a highrisk medicine. Monitoring systems are needed to reduce the morbidity and mortality of patients receiving PN. 